\title{
Lipoatrofia semicircular. A propósito de un caso, en un paciente muy alto
}

\section{Semicircular lipoatrophy. The case of a very tall worker}

\author{
L. Reinoso-Barbero ${ }^{1}$, R. Díaz ${ }^{1}$, M. Piñaga ${ }^{1}$, M.F. Bravo ${ }^{2}$
}

\section{SR. DIRECTOR:}

El objetivo de esta carta es describir un caso clínico de lipoatrofia semicircular (LS) ocurrida en nuestra entidad en 2008 en Madrid y estudiar si sus peculiaridades pueden aportar luz sobre la fisiopatogenia, aún no bien conocida, de esta novedosa enfermedad.

La LS fue descrita en 1974 y se consideró como una atrofia traumática del tejido adiposo subcutáneo. Son lesiones lineales en banda, habitualmente bilaterales y simétricas a 72 centímetros del suelo (la altura estándar del mobiliario de oficina), sin afectación muscular ni de la piel adyacente ${ }^{1}$. También se han descrito casos en el abdomen y antebrazos ${ }^{2}$. En el mecanismo fisiopatogénico clásico parecen intervenir los microtraumatismos repetidos y prolongados en el tiempo, como el apoyo continuado en el borde de la mesa de trabajo. Una nueva hipótesis fisiopatogénica, de cargas electrostáticas y campos electromagnéticos, ha sido recientemente propuesta ${ }^{1}$. Ésta podría explicar los casos epidémicos que han surgido en entornos laborales de empresas que utilizan las pantallas de visualización de datos (PVD) de manera habitual. Según la nueva hipótesis las lesiones serían producidas por las condiciones ambientales de los nuevos edificios altamente tecnificados. Las propiedades bioeléctricas producirían la activación de los macrófagos que secundariamente activarían la secreción de factor de necrosis tumoral alfa (TNF $\alpha$ ) a la hipodermis, produciendo la lesión de los adipocitos por la fagocitosis lipídica. La causa de la LS se cree que es multifactorial. No existe tratamiento curativo, solo preventivo. La adaptación del puesto de trabajo evitando los factores favorecedores conocidos parece ser eficaz en pocos meses ${ }^{1}$. Las acciones correctoras más veces repetidas en la bibliografía son: aumentar la humedad relativa del aire interior del edificio a más del $50 \%$, conectar la mesa a la toma de tierra de protección del circuito eléctrico, colocar mesas de trabajo con bordes anchos, revestir con un aislamiento
1. Medicina del Trabajo. Servicio de Prevención de Riesgos Laborales. Grupo Banco Popular. Madrid. España

2. Medicina del Trabajo. ASEPEYO Mutua de Accidentes de Trabajo y Enfermedades Profesionales de la Seguridad Social. Madrid. España

Recepción: 21 de marzo de 2012

Aceptación provisional: 27 de marzo de 2012

Aceptación definitiva: 27 de marzo de 2012

\section{Correspondencia:}

Luis Reinoso-Barbero

Medicina del Trabajo

Grupo Banco Popular

C/ Juan Ignacio Luca de Tena, 13

28027 Madrid

E-mail: lreinoso@bancopopular.es 
eléctrico el cableado y evitar la presencia de materiales que acumulen electricidad estáti$\mathrm{ca}^{1-3}$. De todos ellos, los que parecen haber dado mejor resultado, según la bibliografía consultada, han sido la combinación simultánea de los tres primeros, al conseguir la remisión completa de las lesiones en el $90 \%$ de los casos en tan sólo 6 meses $^{3}$. La única medida que ha mostrado mejores resultados es la remisión completa del $95 \%$ de los casos tras 1 año de jubilación ${ }^{2}$.

En el caso que nos ocupa nos encontramos con un varón de 38 años, trabajador de banca y aficionado al ciclismo, sin antecedentes personales de interés excepto lumbalgias mecánicas de repetición y su gran altura (1,95 metros). En el momento del diagnóstico llevaba 5 años en la empresa, siempre en el mismo puesto, realizando tareas de oficina sin desplazamientos dentro de su horario laboral. Por las lumbalgias frecuentes y por su gran altura se había adecuado ergonómicamente su puesto de trabajo en el año 2006, elevando 20 centímetros $(\mathrm{cm})$ la mesa, por lo que el tablero quedó a $92 \mathrm{~cm}$ del suelo. En octubre de 2008 acude a consulta por la aparición de una lesión cutánea en la región anterior y lateral del muslo izquierdo de 1 mes de evolución. Al explorar la lesión se observa que es una depresión cutánea en banda semicircular, unilateral, transversal al muslo y a $92 \mathrm{~cm}$ del suelo. Mide $2 \mathrm{~cm}$ de ancho, 10 cm de largo y 3 milímetros de profundidad.
Es indolora, no se observan signos inflamatorios en la lesión y el estado de la piel parece normal. El paciente refiere apoyar los muslos en el canto de la mesa menos de una vez por semana y percibir "chispazos" (descargas electrostáticas) con frecuencia al menos semanal. El resto del examen clínico no presenta alteraciones.

Gracias a que ya se habían dado a conocer casos similares en otras empresas de nuestro entorno, el diagnóstico de sospecha fue LS, por lo que se le remitió a la mutua donde, para descartar afectación cutánea y muscular, le realizaron una ecografía de la región afectada donde se observa una disminución del grosor del tejido celular subcutáneo, posible aumento de la ecogeneicidad de la zona afecta con respecto a la zona anexa, no afectación muscular. Piel y fascia muscular conservadas. No se observan alteraciones musculares y una analítica sanguínea con los siguientes parámetros, todos ellos dentro de la normalidad, hematología, bioquímica general (transaminasas, creatinina, glucosa, colesterol y ácido úrico), con enzimas musculares (lactato deshidrogenasa y creatin fosfato kinasa), y marcadores inflamatorios (proteína $\mathrm{C}$ reactiva y velocidad de sedimentación glomerular). Por lo que se confirma el diagnóstico. No se solicitó ninguna otra prueba adicional. Se tramitó como accidente de trabajo sin baja. El paciente concedió permiso por escrito para registrar gráficamente (Fig. 1) su lesión.

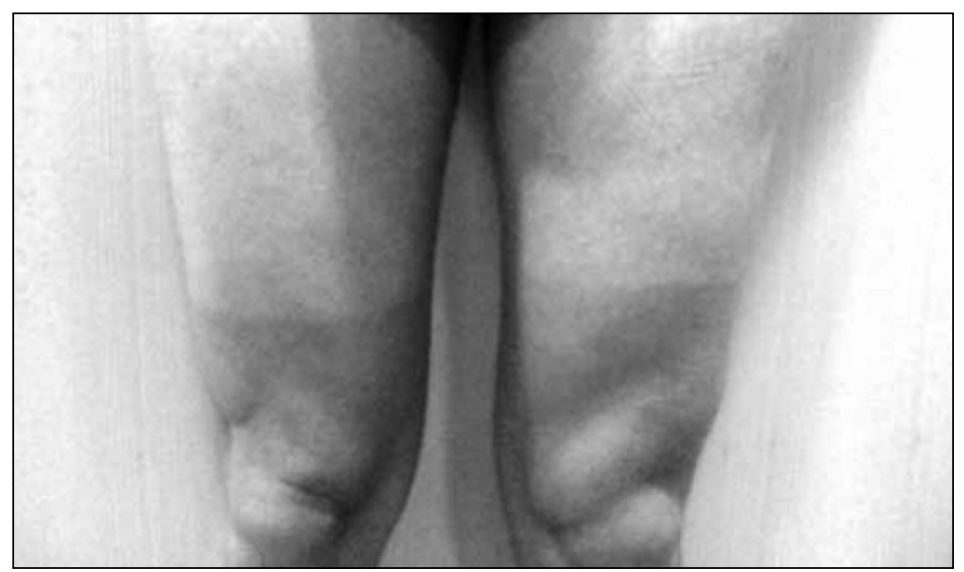

Figura 1. Lipoatrofia semicircular. 
En cuanto a la descripción de las condiciones de trabajo del puesto físico del paciente, podemos destacar que el edificio fue construido en 1983, tiene casi 30 años de antigüedad y coincide en algunos aspectos con la idea de edificio inteligente de la bibliografía consultada ${ }^{1-3}$. Tiene varias características que favorecen la acumulación de cargas electrostáticas: 1) Ambiente caluroso y seco. 2) Las ventanas no son practicables. 3) El suelo está recubierto por moqueta. 4) La silla del trabajador tiene ruedines que friccionan con la moqueta. 5) La silla está forrada por material textil sintético. 6) La mesa es de material sintético y tiene cantos finos y angulosos.

La normativa aplicable en nuestro entorno ${ }^{4}$, obliga a tener unos niveles de humedad relativa superiores al $30 \%$ de humedad y temperaturas que pueden variar desde los $17^{\circ}$ a los $27^{\circ} \mathrm{C}$. Desde diciembre de 2008 hasta octubre de 2009, se tomaron mediciones termo-higrométricas mensuales en el puesto de trabajo afectado y la media aritmética fue de $25,3^{\circ} \mathrm{C}$ de temperatura y $35,3 \%$ de humedad relativa. Es decir, el edificio presenta temperatura y humedad relativa dentro de los valores de referencia pero en los límites bajos de humedad y en los límites altos de temperatura, al igual que muchos de los casos de LS descritos en la bibliografía $^{2-3}$. En nuestro caso no se consiguió modificar la temperatura y la humedad media del edificio. Por lo que se modificaron otras condiciones de su medio ambiente de trabajo que si eran susceptibles de cambio:

A) Se aleccionó al trabajador sobre la importancia de no apoyarse en el canto de la mesa. B) Se le facilitó un reposapiés de material plástico que no alterase la conductividad electrostática (agosto de 2009). C) Se "champuneó" la moqueta de todo el edificio con una sustancia antielectrostática (junio de 2009 y de manera periódica desde entonces). Con esta medida se pretende que el trabajador no genere cargas electrostáticas al friccionar sus zapatos y los ruedines de la mesa con la moqueta. D) Se colocaron alfombrillas de descarga electrostática en zonas comunes de paso (mayo de 2009). E) Se le pasó una encuesta médica trimestralmente donde se le recordaba la importancia de no apoyarse en el canto de su mesa de trabajo.

El trabajador dejo de referir percibir "chispazos" (cargas electrostáticas) y la lesión desapareció definitivamente en octubre de 2009. No ha vuelto a reaparecer hasta hoy (marzo de 2012).

El estudio de este caso parece apoyar a la bibliografía consultada en cuanto que la LS se considera una lesión benigna y reversible, relacionada con los microtraumatismos, cargas electrostáticas y campos electromagnéticos generados en el entorno laboral. Creemos que la utilización frecuente del culot (prenda de vestir propia de los miembros inferiores de los ciclistas, apretada y de fibras sintéticas con roce frecuente al montar en bicicleta) favoreció la aparición de la enfermedad. Que la lesión apareciese a la nueva altura de la mesa muestra la importancia del borde de la misma y su relación con la superficie corporal, por lo que refuerza la teoría fisiopatogénica clásica de la LS. No desmerece, sin embargo, la teoría recientemente propuesta de cargas electrostáticas, ya que la lesión desapareció en menos de un año tras tomar medidas para dejar de apoyar los muslos y para disminuir la generación de cargas electrostáticas.

\section{BIBLIOGRAFÍA}

1. BaChmeyer C, HADDAD A. Lipoatrophia semicircularis of the thighs. Presse Med 2011; 40: 980-981.

2. Curvers B, Maes A. Lipoatrophia semicircularis: a new office disease? 900 cases reported in Belgium. Medecine du Travail et Ergonomie 2004; 41: 53-58.

3. Perez A, Nebot M, Macia M, Panades R. Collaborative Group for Evaluation of LS Outbreak Control Measures. An outbreak of 400 cases of lipoatrophia semicircularis in Barcelona: effectiveness of control measures. J Occup Environ Med 2010; 52: 751-757.

4. Real Decreto 486/1997, de 14 de abril, por el que se establecen las disposiciones mínimas de seguridad y salud e los lugares de trabajo. Boletín Oficial del Estado nº 97 23/04/1997. 